\title{
POBREZA, TRABAJO Y COMUNIDAD EN VASCO DE QUIROGA
}

\section{POVERTY, WORK, AND COMMUNITY IN VASCO DE QUIROGA}

\author{
CECILIA SABIDO SÁNCHEZ JUÁREZ ${ }^{1}$ \\ Universidad Autónoma de San Luis Potosí, México \\ ÉLIDA MARÍA TEDESCO \\ Universidad Autónoma de San Luis Potosí, México
}

Recibido: $14 / 08 / 2019$

Aceptado: 23/09/2019

\section{RESUMEN}

Discutimos algunas tesis económicas presentes en el pensamiento humanista español y su influencia en Vasco de Quiroga. En primer lugar, consideramos su idea de "pobreza", comparada con la de su coetáneo Juan Luis Vives, dado que los hospitales-pueblo estaban orientados principalmente a los pobres; en segundo lugar, la importancia del trabajo y su distribución comunitaria como el elemento clave que retoma de la utopía moreana, relacionadas con las nociones de comercio, dinero y propiedad y; en tercero, la práctica de la "comunidad de bienes" que retoma de la Iglesia primitiva y reconoce como una forma original y propia de la economía indígena originaria.

Palabras clave: comunidad de bienes, humanismo, pobreza, Tomás Moro, trabajo, Vasco de Quiroga.

1 Las autoras son profesoras investigadoras de la Facultad de Ciencias Sociales y Humanidades de la Universidad Autónoma de San Luis Potosí y pertenecen al cuerpo académico de Estudios Decoloniales (UASLP-CA-271). 


\section{ABSTRACT}

We discuss some thesis on economics considered by the Spanish humanist thinkers and how they influenced Vasco de Quiroga. First, we consider his idea of poverty in comparison to that of his contemporary Juan Luis Vives, since town-hospitals were founded mostly to relieve the poor. Then, we consider how did Thomas Moore's view on the importance of work and its communitarian approach inspire his, and its relation with commerce, money and property. Finally, the article briefly studies the concept of "communitarian goods" that Quiroga takes from the ideal of the primitive Church, since he considers it resembles the original indigenous economy.

Keywords: communitarian goods, humanism, Juan Luis Vives, poverty, Thomas Moore, Vasco de Quiroga, work.

\section{INTRODUCCIÓN}

El interés por la economía y su relación con la vida política cobró un nuevo auge a raíz de las lecturas humanistas del siglo $\mathrm{XV}^{2}$. Las nuevas traducciones latinas de fuentes clásicas y las preocupaciones de la vida cotidiana orientaron las reflexiones de la filosofía práctica hacia la consideración de problemas relativos al sostenimiento del hogar, del mercado y la riqueza. Las prácticas comerciales que transformaron la vida de los principados italianos desde Las Cruzadas abrieron nuevos problemas y demandaron respuestas, a veces ya desarrolladas en la tradición y otras que pedían una mayor creatividad. Poco a poco la economía dejó de ser un mero apartado de la Filosofía Política para convertirse en un tema de atención exhaustivo con situaciones particulares, de tal manera que la vida práctica comenzó a disputar a la vida contemplativa su lugar preponderante. Esta tendencia tuvo representación también en el humanismo castellano del Cuatrocientos ${ }^{3}$.

El protagonismo de la vida económica, además, adquirió un apogeo inusitado a raíz de las exploraciones de los navegantes que derivarían eminentemente en el descubrimiento del continente americano. Junto con las reflexiones

2 Al respecto, consultar la recopilación que Eugenio Garin hace de las posturas humanistas relativas al trabajo y la vida económica en El renacimiento italiano (Barcelona: Ariel, 1986), 119-133, donde recoge posturas de Pontano, Collenuccio, Bruno, San Bernardino, Dominici, Nifo y sobre todo de Alberti. Queda para otro momento realizar un estudio sobre estos pasajes.

3 Cf. Juan Miguel Valero Moreno, "Antecedentes y encrucijadas de la vida activa y contemplativa en la Castilla del Cuatrocientos" eHumanista 29, (2015): 32-71. 
políticas, jurídicas y morales que suscitó el encuentro con los pueblos indígenas y su conquista, se multiplicaron también las prácticas económicas y sus cuestionamientos. Por un lado, los nuevos exploradores eran sobre todo comerciantes cuyos principios de conducta no estaban motivados por la fe y el estado ${ }^{4}$, sino por la eficiencia, el sentido común y otros valores como la ambición, la competencia y la ganancia ${ }^{5}$. Sus prácticas distaban mucho de la vida política en que se inscribía la vida de las comunidades feudales: el provecho era propio y se justificaba, en todo caso, por la experiencia de satisfacción del comprador ${ }^{6}$. En consecuencia, no fue fácil tener el control jurídico y moral de los procesos de conquista ni regularlos en torno a los principios de la Corona y de la Iglesia.

Hubo que crear instituciones comisionadas por la Corona para regular la administración hacendaria de las colonias, el comercio con las Indias orientales y occidentales y regular la explotación de sus riquezas, como la Casa de Contratación de Sevilla y el Consejo de Indias ${ }^{7}$. A su vez, los pontífices intervinieron frecuentemente en disputas que no implicaban sólo el control territorial, sino el provecho comercial que de él se desprendía. Además, pasadas las primeras acciones de exploración y conquista fue urgente determinar principios reguladores para justificar la presencia de los conquistadores (en su mayoría, soldados y aventureros que trataban las tierras como propias y a los habitantes como

4 Silvio Zavala, "Marcel Bataillon, Erasmo y España (1950)", en Recuerdo de Vasco de Quiroga (México: Porrúa, 2007), 159.

5 Collenucio, por ejemplo, los llama "filotimos". Advierte que virtud y riqueza no pueden estar juntas y los describe con desprecio: "Y es que no hay nada más inicuo o indigno que aquel que quiere estar siempre en lugar preferente en las bodas, las exequias y los sacrificios, y que, en cambio, en la defensa de la patria, las batallas, las disputas y los juicios, en la eternidad de las obras y, en suma, en las acciones viriles, quiere no sólo ser el postrero, sino que, además, opinando perversamente, estima de gran sagacidad y notoriedad despreciar las ciencias y las buenas artes, y a la vez utiliza todo su estudio y toda su industria, sórdidamente, para acumular bienes, no como patricio civil, sino casi como vil administrador rural". La cita muestra claramente cómo se ven al burgués y al comerciante partícipes de una ética distinta. Pandolfo Collenucio, "Filotimo", en Operette morali, Pandolfo Collenucio y Alfredo Saviotti (Bari: Latersa, 1929): 83-84, citado en Eugenio Garin, El renacimiento italiano, 122.

6 Podría discutirse largamente acerca del provecho feudal, pero desde su concepción está pensado como un pacto social con un provecho compartido por quienes intervienen en el pacto. El señor feudal es visto como un administrador de bienes que debe rendir cuentas al rey y debe usarlos para protección de los siervos. En cambio, el provecho del comerciante es suyo y sólo suyo y debe responder por los gastos de sus viajes y los riesgos propios del tráfico, dejando el resto del beneficio para reinvertirse en nuevas mercaderías y conseguir una posición más estable en la estructura de la vida social. Cf. Miguel Ángel Pérez Priego, "Encuentro del viajero Pero Tafur con el humanismo florentino del primer cuatrocientos", Revista de Literatura LXXIII, 145, (enero- junio 2011): 131-142.

7 Cf. Manuel Casado Arboniés, "Real Provisión de los Reyes Católicos ordenando establecer en la ciudad de Sevilla una Casa de Contratación y promulgando las Ordenanzas por las que ha de regirse". Alcalá de Henares, 20 de enero de 1503, Archivo General de Indias, Patronato, 251. R. 1, f. 14 vto. Cf. Miguel Ángel Ladero Quesada, El primer oro de América: los comienzos de la casa de contratación de las Yndias, 1503-1511 (Madrid: Real Academia de Historia, 2002). 
esclavos). La evangelización fue una motivación peculiar en este camino, pues no podía justificarse sin más la esclavitud de quien es hermano en la fe.

Sin embargo, aún no quedaban claras las motivaciones de las guerras de conquista como causas justas y con ello la legitimidad de otras acciones derivadas de las mismas, que serían ampliamente discutidas en la Escuela de Salamanca; por ejemplo, la apropiación de los bienes de los habitantes y la explotación de sus tierras. Otro tema de discusión fue la demanda hecha a los indígenas, en calidad de perdedores de una guerra, de pagar tributo a los vencedores, bajo una normativa que les era totalmente ajena. Francisco de Vitoria hará frente a estas cuestiones en las relecciones De indis prior y De indis posterior seu de iure belli, con lo cual abrió una serie de tópicos jurídicos y teológicos que darán lugar a la llamada Escuela de Salamanca. En esos tópicos, los temas económicos tienen una profunda relevancia y anteceden en muchas de sus reflexiones a la economía moderna, como han tenido oportunidad de resaltar diversos estudiosos contemporáneos ${ }^{8}$.

No obstante, con el humanismo y la recepción de la filosofía aristotélica en el siglo XV en Salamanca apareció una serie de consideraciones en torno a las reflexiones económicas y las problemáticas de la vida práctica. Éstas debieron influir, directa o indirectamente, en uno de sus posibles e ilustres discípulos: Vasco de Quiroga ${ }^{9}$. Su formación estuvo marcada por las características del humanismo cívico que inundaba las aulas españolas por aquellos años, mismo que habría de inspirar la guerra de las comunidades, poniendo de relieve algunas

8 I.e. José Barrientos García, Repertorio de moral económica (1526-1670), La Escuela de Salamanca y su proyección (Pamplona: EUNSA, 2011); Marjorie Grice-Hutchinson, The School of Salamanca: Readings in Spanish Monetary Theory, 1544-1604 (Auburn: Ludwig Von Mises, 2011).

9 Sobre el alma mater de Quiroga se abren diversas discusiones. Es difícil probar que realmente estudió en Salamanca y se considera también la posibilidad de que estudiase en Valladolid. No hay registros claros y el cruzar referencias de trayectorias entre familiares y amigos no termina por resolver la cuestión. Así lo señala F. Miranda y propone que haya estudiado Artes y Cánones en Salamanca, y que debió culminar sus estudios con la licencia hacia 1520 con casi 30 años cumplidos. Francisco Miranda, Vasco de Quiroga, varón universal (México: Jus, 2007), 10-11. Arce propone también a Salamanca como la institución de formación de Vasco, dada su amistad con Juan de Tavera y su notable predilección por el humanismo, dado que debió ser alumno de Nebrija en aquel tiempo. Pablo Arce Gargollo, Vasco de Quiroga, jurista con mentalidad secular (México: Porrúa, Universidad Panamericana, 2007). Por su parte, Carlos Herrejón Peredo destaca la relación de Quiroga con Bernal Díaz de Luco, miembro del Consejo de Indias y también relacionado con Juan de Tavera, "Introducción" en Vasco de Quiroga, Información en Derecho (México: SEP, 1985), 12-13. Nicolás León se inclina por Valladolid porque en esa ciudad ejerció como licenciado pero tampoco da muchos datos concretos ni considera el episodio africano, Nicolás León, El Ilustrísimo señor don Vasco de Quiroga, primer obispo de Michoacán. Grandeza de su persona y de su obra, (México: Sucesores de F. Díaz de León, 1903), 8. 
ideas republicanas. Estas temáticas y la apertura literaria de Vasco de Quiroga quedan de manifiesto en sus acciones tanto como en sus escritos.

A continuación, desarrollaremos algunos de los elementos económicos presentes en el pensamiento humanista español y su influencia en Vasco de Quiroga. En particular, queremos considerar su concepto de "pobreza", comparado con el de su coetáneo Juan Luis Vives, dado que los hospitales-pueblo estaban orientados a los pobres, una de las preocupaciones del pensador valenciano. En segundo lugar -y como parte de la preocupación por la pobreza-, la importancia del trabajo y su distribución comunitaria como el elemento clave que retoma de la utopía moreana y, en tercero, la práctica de la "comunidad de bienes" que él retoma de la Iglesia primitiva y reconoce como una forma original y propia de la economía indígena originaria. Estos tres elementos, pobreza, trabajo y comunidad, son parte de una visión humanista de la economía que considera al ser humano como un ser comunitario, en vías de mejoramiento a través del trabajo y la consecución del bien común, desde una condición de "pobreza radical". Explicaremos la presencia de estas ideas a continuación, tanto en Vasco de Quiroga, como en su coetáneo Juan Luis Vives.

Aunque Vasco de Quiroga nunca hace mención explícita de la obra de Vives, la comparación pretende establecer que existió un contexto ideológico y cultural común en el primer tercio del siglo XVI. En cambio, Vasco sí abunda en la influencia que tuvo Tomás Moro en sus ideas. La Utopía se publicó en 1517, diez años antes que el Tratado sobre el Socorro de los Pobres de Vives y éste, a su vez, diez años antes de las acciones y las obras de Vasco de Quiroga. Puede especularse que Vasco de Quiroga - por lo general bien enterado de las obras de su tiempo-conociera bien el pensamiento del valenciano y no lo citara, dadas las acusaciones de luteranismo que rodeaban la obra de Vives y su oscilante prestigio en el ánimo español, dado su pasado judío. Sin embargo, todas estas son suposiciones y sólo podemos afirmar la correlación temática en el humanismo de su tiempo.

\section{POBREZA}

Como se sabe, apenas llegar a tierras americanas, Vasco de Quiroga propuso una alternativa en la creación de asentamientos para la evangelización indígena. Su propuesta consistió en lo que él llamó "hospitales-pueblo". Se trataba de una institución de asistencia peculiar, conformada por familias de indios que vivirían bajo un régimen político autónomo -y en cierto modo igualitario-, 
dedicado al trabajo, el estudio del catecismo y el ocio organizados. Para la planeación de estos pueblos se inspiró en la Utopía de Tomás Moro ${ }^{10}$.

Tanto en la Información en Derecho ${ }^{11}$ como en las Reglas y Ordenanzas para el gobierno de los Hospitales de Santa Fe en México y Michoacán ${ }^{12}$, Vasco de Quiroga se refiere a los destinatarios de su fundación como "pobres". De sus textos y su práctica se desprende inevitablemente un acusado paternalismo y pareciera que Quiroga llama indistintamente pobres a los indios, cual sea su posición. Es indispensable comprender el contexto en que surgen tanto las fundaciones como los escritos del madrigalense para poder analizar el concepto de pobreza que utiliza.

En primer lugar, hace falta destacar que Vasco de Quiroga se refiere al término "pobreza" de manera análoga y habrá que distinguir en su obra varios sentidos: a) uno general, más teórico y relativo a la natural condición humana de necesidad; b) uno cristiano o moral, relativo a las virtudes de desapego, sobriedad y simplicidad, y c) otro propiamente económico en que se refiere a los más necesitados en las estructuras sociales de América. Habrá que contextualizar también qué se entiende por pobreza en el sentido del humanismo cristiano y cómo influye este concepto en la forma de Quiroga de tratar y recibir a los indígenas pobres, que serán los principales destinatarios de los hospitales-pueblo.

No puede dejarse de lado la ineludible influencia del concepto de pobreza franciscana en su tiempo: desde el mismo cristianismo, especialmente a partir de Francisco de Asís y los muchos avatares de las órdenes franciscanas, se discutió mucho sobre los sentidos de pobreza durante la Baja Edad Media. Más allá de una condición desfavorecida, la pobreza se concibe como una virtud

10 Este tema ha sido ampliamente expuesto por Silvio Zavala, Ideario de Vasco de Quiroga (México: El Colegio de México, 1941); Mariano Cuevas, Historia de la Iglesia en México, Tomo I, 1511-1548 (México: Patria, 1946); Herrejón Peredo, "Introducción”; Miranda, Vasco de Quiroga, varón universal; Arce Gargollo, Vasco de Quiroga, jurista con mentalidad secular; Antonio Arriaga Ochoa, "Vasco de Quiroga, fundador de pueblos", Estudios de Historia Novohispana 1, no. 1 (1966): 149-156; y en general, autores como Rafael Moreno, Anthony Pagden, J. C. Davis, Nicolás León, Juan Benito Artigas Hernández, Isidro Castillo, Paz Serrano, Fintan Warren, Benedict Warren, B, y un largo etcétera. Además, Arce ofrece en su citado libro una generosa bibliografía temática.

11 Vasco de Quiroga, Información en Derecho, versión de Carlos Herrejón Peredo, (introducción y notas), (México: Cien de México-SEP, 1985). Tomo la numeración propuesta en esta edición.

12 Tomo la versión moderna presentada por Arce Gargollo, "Reglas y ordenanzas de un Pueblohospital" en Vasco de Quiroga, jurista con mentalidad secular, 139-154. Citaré principalmente su enumeración. También retomo con él la versión de Rafael Aguayo Spencer, Don Vasco de Quiroga. Pensamiento jurídico, Antología (México: Porrúa, 1979). El segundo número que aparezca entre paréntesis corresponde a esta versión. 
evangélica que pone en segundo plano la realidad material para privilegiar la riqueza espiritual y el vínculo del pobre con Cristo y su reino.

No obstante, y dejando de lado la práctica mística, la pobreza fue considerada un problema moral y cívico creciente durante el Renacimiento. De ello da cuenta la solicitud que la ciudad de Brujas hizo al humanista valenciano Juan Luis Vives de la redacción de un tratado que iluminara a la ciudad sobre el modo en que debía atenderse el problema de la mendicidad atendiendo a la justicia, a la caridad cristiana y a la dignidad humana ${ }^{13}$. De esta solicitud surgió De subventione pauperum, o Tratado del socorro de los pobres, publicado en $1526^{14}$. En esta obra, Vives estudia el origen de la necesidad y la miseria de todo ser humano, que para él radica en la naturaleza caída por el pecado original. El orden de la constitución humana queda invertido y su naturaleza dañada de tal manera que su vida prácticamente parece un estado de "muerte", una lucha entre el alma y el cuerpo que sólo puede aspirar al éxito si éste se une a otros para formar familia y ciudad; por eso dice en su dedicatoria: "también porque como haya sido el origen de todas las ciudades, con el fin de que cada una de ellas fuera un lugar en donde, con dar y recibir beneficios, y con el auxilio recíproco, se aumentase la caridad, y afirmase la sociedad de los hombres"15. También menciona el humanista que con esta intención se crea el dinero, para realizar los intercambios necesarios.

No obstante, señala Vives que algunos hombres pretendieron vivir del trabajo de otros, ya fuera por pereza, arrogancia y miseria humana, por enfermedad, por perder la guerra, o por mala administración o mala suerte. Además de las causas exteriores considera las interiores: la falta de o la mala educación, la

13 Sebastián Solanes, "La aportación de Juan Luis Vives al estudio de la pobreza. Del socorro de los pobres a la aporofobia", Vivesiana 3 (2018): 80.

14 La dedicatoria de Vives declara sus intenciones: "A vosotros dedico esta obra, ya porque os esmeráis en hacer bien y aliviar a los miserables, de que da bastante testimonio la muchedumbre de pobres que concurre de todas partes aquí, como a refugio siempre prevenido para los necesitados, ya también porque como haya sido el origen de todas las ciudades, con el fin de que cada una de ellas fuera un lugar en donde, con dar y recibir beneficios, y con el auxilio reciproco, se aumentase la caridad, y afirmase la sociedad de los hombres, debe ser particular desvelo de los que gobiernan cuidar, y poner todo esfuerzo en que unos sirvan a otros de socorro, nadie sea oprimido, nadie injuriado, nadie reciba daño injusto, y que al que es más débil asista el que es más poderoso, y de esta suerte la concordia del común, y congregación de los ciudadanos se aumente cada día en la caridad, y permanezca eternamente". Juan Luis Vives, Tratado del socorro de los pobres, trad. Juan de Gonzalo Nieto, Clásicos de Historia, Martínez J., (comp.). http://clasicoshistoria.blogspot.com/2017/09/juan-luisvives-tratado-del-socorro-de.html. Consultado el 3 de abril de 2019.

15 Ídem. 
corrupción y la mala disposición, de manera que los seres humanos por causa de la soberbia terminan dependiendo del auxilio de los otros ${ }^{16}$.

Concluyamos pues que todo aquel que necesita de la ayuda de otro es pobre, y menesteroso de misericordia, que en griego se llama limosna, la cual no consiste sólo en distribuir dinero como el vulgo piensa, sino en cualquiera obra por cuyo medio se socorre la miseria humana ${ }^{17}$.

En suma, para Vives la pobreza es ocasión de hacer el bien, pues no hay mayor beneficio que "coadyuvar uno a la virtud del otro". Todos necesitan ayuda, de una manera $u$ otra, y hay muchas formas de hacer el bien en la vida política que son más adecuadas y beneficiosas que la del dinero. Así como el pecado causa la pobreza, también causa el egoísmo de quienes niegan el socorro al necesitado ${ }^{18}$. Como recuerda Sebastián Solanes en su análisis de la pobreza en Vives:

(...) la ciudad debe ser un intercambio de los beneficios y la reciprocidad de los auxilios, a fin de que puedan consolidarse las necesarias ayudas de caridad a los ciudadanos más desfavorecidos, que deben ser los que caractericen una sociedad que quiera llamarse humana. ${ }^{19}$

Así pues, la pobreza vista desde el humanismo es, por un lado, una consecuencia de la naturaleza caída del hombre y, por otro, un asunto que compete remediar a la comunidad ya sea por medio de las disposiciones de los gobiernos o por medio de la caridad de los propios ciudadanos. Quizá sea mejor reformular y decir que, precisamente de la caridad nativa en el alma de los ciudadanos surgen los gobiernos, cuya natural disposición debe ser la de remediar las necesidades de los débiles. Es, en otras palabras, un asunto concreto de humanismo cívico, más relacionado con la experiencia comunitaria de la virtud que con la disposición efectiva de dinero.

La forma adecuada de superar la pobreza -y así lo manifiesta también Moro- es el trabajo ${ }^{20}$. Para que este remedio sea verdadero debe estar fortalecido por la instrucción, que no es sólo la preparación técnica sino la formación cívica

16 Vives, Tratado del socorro de los pobres, 4-6.

17 Ibíd., 6.

18 "Fuera de esto, si socorriéramos a los pobres con prontitud y a tiempo, sin duda se seguiría el grande y público bien de que con la condición y estado de sus cosas mudaran ellos sus costumbres, pero en el día dejamos a los mendigos que se pudran en su necesidad; ¿pues qué pueden sacar ellos de sus inmundas miserias sino todos los vicios que ya hemos referido? Por eso sus culpas son miserias humanas y de algún modo necesarias, pero las nuestras son voluntarias, libres y casi diabólicas..." Vives, Tratado del socorro de los pobres, 16.

19 Solanes, "La aportación de Juan Luis Vives al estudio de la pobreza", 82.

20 Utopia, I, 5-3. 
que integra al individuo a la vida política. Tanto la Utopía de Moro como los hospitales-pueblo de Vasco de Quiroga coinciden con este doble remedio: trabajo e instrucción cívica ${ }^{21}$.

Ahora bien, ¿a qué sentido del término "pobreza" responde la creación de los hospitales-pueblo? ¿por qué llama Quiroga pobres a los nativos michoacanos en la Información en Derecho? En la redacción de su Testamento manifiesta claramente su motivación:

Movido de devoción y compasión de la miseria e incomodidades grandes y pocas veces vistas ni oídas que padecen los indios, pobres, huérfanos y miserables personas, naturales de estas partes, donde por ello muchos de los de edad adulta se vendían a sí mismos y permitían ser vendidos y los menores y huérfanos eran y son hurtados de los mayores y vendidos, y otros andan desnudos por los tianguices aguardando a comer lo que los puercos dejan y esto además de su derramamiento grande y falta de Doctrina Cristiana y moral exterior y buena policía, fundé y doté a mi costa y de mis propios salarios, con el favor de Dios nuestro Señor y de su Majestad el Emperador y Rey don Carlos nuestro Señor, dos Hospitales de indios que intitulé de Santa Fe (...) para sustentación y doctrina, así espiritual como moral exterior y buena policía de indios pobres y miserables personas, pupilos, viudas, huérfanos y mellizos, que dicen mataban las madres por no los poder criar por su gran pobreza y miseria; y estos todos que sean ciertos y perpetuos y tantos en número cuantos cada uno de los dichos hospitales puedan cómoda y buenamente sustentar...22

El texto de Vasco de Quiroga deja entrever, en primer lugar, el tercer sentido de pobreza que mencionamos antes y se refiere a las penosas necesidades que sufrían muchos indios, la mayoría a consecuencia de la destrucción de su estilo de vida y la imposición rapaz de los primeros encomenderos y miembros de la primera Audiencia, a quienes Quiroga había tenido que auditar, precisamente por estas razones. En efecto, esta primera misión como oidor le permitió ver en profundidad las terribles consecuencias que los vicios de los conquistadores habían tenido sobre la población nativa de América. No diré que Vasco de Quiroga se dé a la tarea de condenar y criticar la conquista, nunca lo hizo al modo ni con la vehemencia de Las Casas. Su aproximación fue más inmediata y práctica: poner una solución que le permitiera cumplir con su misión

21 Sobre esto hemos tratado previamente en Cecilia Sabido, "El ideal humanista de mejoramiento humano y su influencia en Vasco de Quiroga”, Metafisica y persona 21 (2019): 183-197.

22 Versión de Rafael Aguayo Spencer, Don Vasco de Quiroga. Pensamiento jurídico, Antología (México, Porrúa-UNAM, 1986). 
evangelizadora y paliar los daños causados, ya por los abusos de los encomenderos, ya por los modos de vida que los indios tenían, que le parecieron bárbaros e ignorantes ${ }^{23}$.

Resulta intrigante, sin embargo, que se refiera siempre a los habitantes de los hospitales como "indios pobres". De hecho, estatutariamente afirma en las Reglas y ordenanzas que "El hospital está constituido por los pobres" 24 y más adelante repite "los padres de cada familia de todos los pobres del hospital deberán quedar divididos en cuadrillas" 25 . En fin, los ejemplos son muchos y sugieren que la condición de "pobreza" es esencial para formar parte de la comunidad $^{26}$. Aquí conviene aplicar el primer sentido de pobreza que hemos señalado previamente, el hecho de que los miembros de una comunidad política se necesitan unos a otros. Nadie puede alcanzar la virtud solo. Esta es una de las principales causas que mueven a Vasco de Quiroga a proponer la creación de estos pueblos como remedio de la disgregación en que los nativos han incurrido para escapar de la esclavitud: la necesidad de vivir en comunidad. Así lo manifiesta en la Información en Derecho ${ }^{27}$.

Aunque pudiera admitirse una interpretación de soberbia y superioridad en Vasco de Quiroga sobre los indios, sobre todo con fundamento en el manejo del término en la Información en Derecho, es igualmente congruente la visión

23 Hoy día puede juzgarse con dureza la aproximación de Quiroga a la realidad nativa. Lo cierto es que, entre sus coetáneos, la suya fue una de las aproximaciones más respetuosas y conscientes de la dignidad de nos pobladores originarios. Ya el testimonio de Cabrera muestra cómo evangelizó por medios atractivos, invitando y no imponiendo (cf. Arce, "Testimonio de Cristóbal Cabrera", en Vasco de Quiroga, jurista con mentalidad secular, 123-137), y sin pretender una exégesis de sus medios, no puedo menos que subrayar que como humanista veía en la condición de los indios un estado bárbaro y un llamado connatural a la virtud. Sin duda, ninguna de estas visiones escaparía a una crítica del eurocentrismo conquistador, pero al considerar los matices y vertientes de un planteamiento, el de Quiroga destaca por poner atención en "el otro". También puede citarse el documento que recupera Cuevas a este respecto: "Será una grande obra pía y muy provechosa y satisfactoria para el descargo de las conciencias de los españoles que acá han pasado, que se cree que mataron e fueron causa de ser muertos en las guerras y minas los padres y madres de tales huérfanos y de haber quedado así pobres que andan por los tiangues a buscar de comer lo que dejan los puercos y los perros, cosa de gran piedad de ver, y estos huérfanos y pobres son tantos que no es cosa de se poder creer si no se ve". Carta al Consejo de Indias, fechada el 14 de agosto de 1531. Citada en Cuevas, Historia de la Iglesia en México, 354.

\section{Ordenanzas 8.}

25 Ordenanzas 11.

26 También Cabrera, en su testimonio, que resulta bastante esclarecedor del modo en que se llevan a cabo algunas prácticas, señala: "Mas en aquellos dos pueblos donde eran catequizados los catecúmenos y adonde confluían los pobres (porque pobres eran casi todos los indios plebeyos, fuera de algunos principales que esquilmaban al común del pueblo), a todos se les daba allí hospedaje y alimentos, hasta tanto que, instruidos en las cosas de la Fe y bautizados regresaban a sus casas". Testimonio de Cabrera, apéndice I, § 6, 127.

27 Información en Derecho, II, 20 y III, 31. 
humanista del ser humano como naturaleza caída, ser necesitado que carece de los medios básicos para vivir la virtud: una comunidad en la que todos hagan el bien mutuamente y la instrucción básica para vivir en equidad y justicia. Ahora bien, para Vasco de Quiroga, la mayoría de estas virtudes cívicas están ya presentes en las costumbres de los indígenas y sólo es necesario restaurar la posibilidad de vivir en comunidad, bajo el modelo utopiano que, como hemos señalado en otro lugar, bien pudo inspirarse en las prácticas comunitarias america$\operatorname{nas}^{28}$.

En Vasco de Quiroga, todo el plan de la vida comunitaria está encaminado a promover la naturaleza humana de los indios, no porque sean inferiores a los españoles sino para preservar la pureza de sus costumbres y evitar la contaminación de los vicios europeos ${ }^{29}$. Pero esta promoción implica superar la condición de pobreza, tanto en el sentido práctico - la condición en que viven- como en el antropológico -el crecimiento en virtud que requiere todo ser humano-. Por otro lado, Quiroga siempre considerará como ventajosa la sobriedad a la que están acostumbrados los indios, que corresponde al segundo sentido de pobreza y que se aproxima al sentido más franciscano - comparable con la iglesia primitiva- y virtuoso de vivir sin lujos, desapegado de las riquezas y bienes particulares. Esta comunidad está llamada, en su opinión, a constituir la "nueva iglesia renaciente" 30 . El medio eminente para combatir la pobreza en la comunidad es el trabajo. Sobre esto hablaremos a continuación.

\section{TRABAJO}

En Información en Derecho, Vasco de Quiroga extiende y detalla las razones que expuso en su Parecer como oidor de la segunda Audiencia, una especie de diagnóstico tras una exhaustiva auditoría a las acciones de la primera. Si bien el documento oficial se perdió, se conserva este otro, escrito a modo de carta, que recoge y detalla lo que más le preocupa: por un lado, explica por qué dictaminó que el esclavismo es impropio e injusto en tierras mexicanas y no debe confundirse con las relaciones de servidumbre voluntaria que se practicaban en el mundo prehispánico ${ }^{31}$; por otro, advierte el peligro en que se encuentra la

28 Cf. Cecilia Sabido, "El programa humanista en la utopía práctica de Vasco de Quiroga", en Tomás Moro y Vasco de Quiroga, Utopías en América, ed. Virginia Aspe (Pamplona: Pensamiento Medieval y Renacentista EUNSA, 2018), 115-135.

29 Silvio Zavala, "La utopía de Tomás Moro en la Nueva España", en Recuerdo de Vasco de Quiroga, 13.

30 Información en Derecho, III, 241. Ver también III, 238.

31 Silvio Zavala, Ideario de Vasco de Quiroga, 11-28. 
población indígena a raíz de los excesos de los encomenderos, de tal manera que propone la creación de una comunidad que no sólo sirva para evangelizar a los indios, sino para promoverlos a través del trabajo, la educación y la convivencia y evitar así la condición de pobreza en la que estaban viviendo. El proyecto de los hospitales-pueblo tiene tres fuentes originarias: la Utopía de Tomás Moro, la imagen de la edad dorada de los textos clásicos -principalmente en las Saturnales de Luciano y la Égloga IV de Virgilio-, y el testimonio de las comunidades protocristianas del Nuevo testamento. En estas tres inspiraciones se da un valor ambivalente a la idea de trabajo.

El candor de los indígenas que Quiroga admira adolece de ocio y pereza ${ }^{32}$. La "nueva edad de oro" como la que Virgilio anuncia en la Égloga IV implicaba asimismo un nuevo advenimiento de Saturno ${ }^{33}$ donde sin esfuerzo se obtendrían los frutos de la tierra ${ }^{34}$. Quiroga está convencido, sin embargo, de que la industria es la virtud que falta a los naturales americanos, porque ella los protege de las adversidades y la miseria, y además, les permitirá cumplir con los tributos que deben sin perder su sustento ${ }^{35}$. El modelo de Tomás Moro que Quiroga retoma en su proyecto es una superación de la edad dorada a través del trabajo, sin perder la capacidad de vivir en comunidad, de despreciar la apropiación individual de los bienes materiales y de vivir en la humildad y la mansedumbre

32 Así lo acusa gravemente en las Ordenanzas donde parece subrayar el ocio y la pereza como males propios de la naturaleza indígena: "Y ha de ser no rehusándole, ni escondiendo ni os apartando, ni excusando de él vergonzosa, perezosa y feamente, como lo soléis hacer..." Y subraya más adelante: "Y sois muy defectuosos por lo que os vienen grandes e irremediables males, inconvenientes e incomodidades, así a vuestros cuerpos como a vuestras ánimas como a vuestras personas y bienes temporales". Ordenanzas, 73

33 Redeunt saturnia regna. III, 237. Égloga IV, 6

34 "Vuelve ya también la Virgen, vuelve el reinado de Saturno; una nueva descendencia baja ya de lo alto de los cielos. Tú, casta Lucina, sé propicia al niño que ahora nace, con él la raza de hierro dejará de serlo al punto y por todo el mundo surgirá una raza de oro. Tu Apolo reina ya." IV, 5-10. Dice también: "Mas para ti, ;oh niño!, la tierra sin cultivo alguno derramará en primicias" IV, 19; "Mas luego, cuando la edad ya fortalecida te haya convertido en hombre, el nauta mismo abandonará la mar y el pino marinero no trocará las mercancías; toda tierra producirá de todo. El campo no consentirá los rastros ni la hoz la viña; y por su parte el robusto labrador desuncirá los toros" IV, 37-40. Por su contenido, la Bucólica o égloga IV fue considerada con frecuencia una suerte de "premonición" del Cristianismo. En todo caso, anuncia una nueva era de paz, sin dificultades ni esfuerzos. Cf. Plubio Virgilio Marón et al., Bucólicas, Geórgicas, Apéndice virgiliano, trad. T. Recio García (Madrid: Gredos, 1990), 187-190.

35 "Y darles tal orden y estado de república y de vivir, en que se pierdan los vicios y se aumenten las virtudes, y no pueda haber flojedad ni ociosidad ni tiempo perdido alguno que les acarree necesidad y miseria, y pierdan la mala costumbre de este ocio dañoso en que están criados y acostumbrados, y de manera que no lo sientan ni pierdan, como dicho es, hora ni tiempo ni la gasten mal gastada ni la empleen mal empleada, y se ordene en todo de manera que para sí les baste poco, y para cumplir con las cargas que han de llevar y tributos que han de pagar para la sustentación de todos, les sobre mucho, y juntamente con esto de su buena voluntad y simplicidad no pierdan nada". Información en Derecho, III, 246. 
del protocristiano, en un advenimiento de lo que Vasco de Quiroga considera como la Iglesia renaciente en el Nuevo Mundo ${ }^{36}$.

A Vasco de Quiroga le preocupa especialmente que la mala comprensión de las condiciones laborales en la colonia termine por costar la vida a los pobladores del continente, pues desacostumbrados al ritmo de trabajo que los encomenderos les imponen, se ven a sí mismos explotados, herrados, fatigados, incapaces de cumplir con los lujos y exigencias de los conquistadores ${ }^{37}$. Su razonamiento es práctico: piensa que la industria en el contexto de la organización comunitaria no sólo les permitirá vivir, sino también satisfacer sus necesidades y las exigencias del tributo, tener suficiente abundancia para vivir bien y hacerlo en paz y concordia ${ }^{38}$. De esta manera alcanzan no sólo un sistema de supervivencia en un sentido económico, sino una comunidad política en el sentido pleno de la palabra.

La Utopía de Moro se basa fundamentalmente en la repartición equitativa de trabajo, propiedad y ocio entre los miembros de la comunidad. Se destaca la importancia del trabajo comunitario porque a través de éste se procura la virtud y se remedian los vicios ${ }^{39}$. Vasco de Quiroga ve en el trabajo una vía de mejoramiento humano, capaz de fortalecer el vínculo social y de rescatar, de un modo concreto, a los indios de su situación presente con dignidad y autonomía: la implementación de los hospitales-pueblo conseguiría aumentar la producción, no sólo para satisfacer la necesidad presente y futura de los integrantes de la comunidad (incluido el sacerdote que la preside) sino también para poder pagar el lastimoso tributo sin esfuerzo ni pérdida de su valía ${ }^{40}$.

La idea de Vasco de Quiroga, inspirada por Moro, consiste en organizar las actividades de tal manera que se trabaje poco y se tenga el suficiente rendimiento para que aún quede espacio para el estudio, el ocio y la vida comunitaria. $\mathrm{Si}$ bien propone el trabajo como solución, no se trata de una explotación del hombre en aras de la productividad, sino una auténtica "oikonomía": la vida está

36 Información en Derecho, III, 241.

37 Información en Derecho, III, 37-39.

38 "Que bastante sea para llevar, sufrir y sustentar mucho tiempo las cargas incomportables nuestras y de nuestra soberbia y grand cobdicia y presunción, y que por no querer mirar a lo poco a que bastan y pueden, no nos contentamos con poco, sino que siempre les pedimos lo que ni tienen ni pueden. Y así nos dan los hijos, las vidas y la sangre y todo por ser gente tan sin arte para ello". Información en Derecho, III, 266

39 "Sean cada día menos los que viven en la ociosidad; que se vuelvan a cultivar los campos, y que vuelva a florecer la industria de la lana! Sólo así volverán a ser útiles toda esa chusma que la necesidad ha convertido en ladrones o que andan como criados o pordioseros a punto de convertirse también en futuros ladrones." Utopía I-5, 3 .

40 Información en Derecho, III, 246. 
administrada, limitada y organizada con miras a llevar una existencia cualitativamente feliz y claramente autosuficiente.

De hecho, los hospitales-pueblo no sólo llegaron a producir lo necesario para la subsistencia de sus participantes, sino que sostenían otras fundaciones de Quiroga, como el Colegio de San Nicolás. Así queda detallado en su testamento, donde destina el equivalente al valor de 300 ducados anuales producidos por los Hospitales de Santa Fe a dicho colegio ${ }^{41}$. Además, los hospitales-pueblo entraron a formar parte de una red de producción que fue estableciendo Vasco para otros pueblos donde las condiciones eran menos precarias, pero donde aún se carecía -al menos en su opinión- del hábito productivo. Para ellos, Vasco de Quiroga ideó un sistema de producción artesanal de bienes y servicios que eran intercambiados a modo de trueque, sin necesidad de dinero ${ }^{42}$. Al respecto, dice Miranda:

Fue Quiroga, según lo conocemos, un estimulador decidido de esas formas de economía que hacían del comercio un vehículo importante para mejorar la condición humana de las poblaciones, fomentaba congregación y aunque sin duda nada inventó, sí puso especial acento en la especialización de cada comunidad, para ese intercambio de bienes en los mercados ${ }^{43}$.

Así, según Miranda, en la "eutopía comunitarista" de Vasco de Quiroga, el comercio es una acción social, propia de un humanismo cívico.

Si bien este vasto proceso creativo en Michoacán tuvo su origen en las fundaciones de Fray Juan de San Miguel -quien estableció las primeras huatáperas y los pueblos de Tancítaro, Charapan, Periban y Uruapan-, Vasco de Quiroga retomó aquel impulso de modo que dio forma y "sentido económico, social y agrario" al estilo de vida y la fuente de riqueza de muchos pueblos tarascos ${ }^{44}$. Después de enlistar sus especialidades artesanales, Arriaga recuerda que además Quiroga fijó un día de mercado para cada pueblo, de manera que la actividad económica fluyera naturalmente ${ }^{45}$.

41 Testamento, 28-29.

42 Miranda, Vasco de Quiroga, varón universal, 92-93; ver también Juan José Moreno, Fragmentos de la vida y virtudes del Ilustrísimo don Vasco de Quiroga (México: Imprenta del Real y más Antiguo Colegio de San Ildefonso, 1766) 140.

43 Ídem, 93.

44 Campos calcula hasta 200 pueblos políticamente autónomos. Cf. Carlos Alberto Campos, "Carlos V, Tomás Moro y Vasco de Quiroga", XXII Coloquio de Historia Canario-Americana (2016), XXII-072, 1-11 http://coloquioscanariasmerica.casadecolon.com/index.php/aea/article/view/10017. Consultado el 15 de abril de 2019.

45 "Santa Clara del Cobre fue lugar para los cobreros; la cerámica policroma se trabajó en Tzintzuntzan, Patamba, Capula, Panícuaro y Santa Fe de la Laguna; las bateas y baúles pintados, en 
Al igual que Moro, en sus escritos Vasco de Quiroga critica el dinero y el modo en que ha dejado de ser un instrumento de intercambio para convertirse en la meta de la vida económica, de tal manera que, si en la Utopía se ridiculiza su valor, en el hospital-pueblo se conserva únicamente como un medio de intercambio con el mundo exterior - para el pago de tributo cuando no se recibe en especie o para realizar alguna diligencia necesaria para el pueblo- pero carece de utilidad y sentido en la dinámica económica de la comunidad. Para él, esto es posible porque las mismas costumbres indígenas tienen el dinero en poco:

...sin tratar moneda entre sí y con grande menosprecio del oro y de la plata, sin aprovecharse del uso ni aprovechamiento dello para más de solamente andar galanes en sus fiestas, hasta que los españoles vinieron, que por tenerlo ellos en tanto, ya lo van teniendo éstos en $\operatorname{algo}^{46}$.

En cambio, toda la riqueza producida y poseída por la comunidad se aquilata en los mismos bienes ${ }^{47}$. Incluso cuando habla de la "caja de tres llaves" que está a cargo del rector, el principal y un regidor, no habla de "dinero" como su contenido sino de "todo lo demás que sea necesario guardarse" ahorro pensando en la previsión, pero no en la especulación en modo alguno:

Y porque haciéndolo así, pocas veces o ningunas os veréis en necesidad, antes vosotros podréis socorrer a muchos necesitados y descuidados, como siempre lo haréis y debéis hacer, con parecer del Rector y Regidores, multiplicando

Cucupao (Quiroga); en Teremendo y Azajo nació la industria de la curtiduría; las esteras de tule se fabricaron en Zirándaro e Ihuatzio; la carpintería floreció en Cuanajo; en Paracho se hicieron los instrumentos musicales y se perfeccionó la tintorería; en San Felipe, la herrería; en San Juan Parangaricutiro se produjeron las colchas hechas con el viejo sistema de la patacua o telar primitivo de los tarascos; los deshilados, en Aranza; en Nurio se desarrolló la sombrerería. En Pátzcuaro brilló el artesanado en todos sus aspectos; fue el centro de sus maestros. Después de la desaparición de la Casa de Altos Estudios en Tiripitio, en Pátzcuaro se encontraban los artistas que hicieron los Cristos de caña; allí se fabricaban los instrumentos musicales de latón, especialmente las chirimías, las telas, las cobijas; se practicó la platería y la industria de las lacas pintadas con pincel, en las cuales se introdujo el oro como motivo decorativo, y finalmente, se dominó la técnica del vidrio soplado". Sería largo el hablar de las antiguas industrias que autorizaron el elogio del cronista Larrea: "Son eminentes (los indígenas) en todos los oficios, de tal manera que sus curiosidades han recorrido todo el mundo con aplauso general". Arriaga, "Vasco de Quiroga, fundador de pueblos", 7.

46 Información en derecho, 240.

47 Miranda incluso refiere que el trueque sobrevivió en la práctica económica michoacana hasta nuestros días: "Sobrevivió el trueque que ha tardado mucho en ser sustituido por la moneda y aún no lo logra totalmente. No sabemos hasta cuándo hayan persistido el cacao y las mantas como valores". Miranda, Vasco de Quiroga, varón universal, 93.

48 A este respecto, Cuevas cita una carta inédita, dirigida a unos habitantes de Santa Fe en México donde determina qué hacer con la caja y cómo restituir un dinero que había tomado, a la vista de todos para que no haya dudas. Cf. Cuevas, Historia de la Iglesia en México, Tomo I, 357-358. 
el depósito de sobras por más años y no solamente por uno, si cosa conveniente fuere ${ }^{49}$.

Quitando valor práctico al dinero, Vasco de Quiroga pretende instaurar una vida económica alternativa, simultánea pero incontaminada por la corrupción de sociedad conquistadora, las tres bestias que, según declara en las Ordenanzas, se deben evitar: "Hay que ponerse fuera de peligro de las tres fieras bestias que todo en este mundo lo destruyen y corrompen, que son soberbia, codicia y ambición, de las que os habéis y deseamos mucho guardar y apartar" ${ }^{20}$. Finalmente la riqueza pertenece a la comunidad y se vive precisamente en la plenitud de la vida práctica. El dinero sólo materializa parte de esa riqueza para los fines del otro mundo. El origen de la riqueza, para Vasco de Quiroga y para Tomás Moro se encuentra, por lo tanto, en el valor del trabajo ${ }^{51}$. En lugar de depender de la sola naturaleza, la Utopía propone una sociedad que mejora gracias al trabajo, y a que se realiza en común y para la comunidad ${ }^{52}$.

Otro elemento económico relevante y relacionado con la pobreza es el sentido de propiedad. Vasco de Quiroga no elimina la propiedad, porque es para él muestra de madurez y autonomía. Sin embargo, ésta debe ser comunitaria en el régimen de los hospitales-pueblo. La propiedad privada, tal y como la menciona, es fuente de vicio y ambición. Al respecto da una clara instrucción en las Ordenanzas:

Porque si fuese de otra manera, se perdería esta buena obra y limosna de indios pobres y huérfanos, pupilas y viudas y miserables personas fácilmente, (...) apropiándolo cada uno para sí lo que pudiese y sin cuidado de sus prójimos, como es cosa verosímil que sería, y se suele hacer, por nuestros pecados, y por falta de semejante policía y concierto de República, que es procurar lo propio y menospreciar lo común, que es de los pobres ${ }^{53}$.

49 Ordenanzas 44, 20.

50 Ordenanzas 5, 6.

51 Al respecto recomiendo el excelente artículo de Manuel Méndez Alonso, "La discusión sobre Vita Activa, Nobilitas y Libertad en la Utopía de Tomás Moro", Revista Portuguesa de Filosofia 67, no. 2 (2011): 355-376.

52 Esta sola idea implica muchos retos, dado que las diferencias sociales, en su mayoría, se desprenden de la división del trabajo. Cuando la sociedad se organiza con tal rotación que permita a todos ocupar las mismas actividades por turnos, las diferencias que se desprenden del trabajo disminuyen, todos aportan en equidad y por lo tanto merecen participar de los bienes de la comunidad en igualdad.

53 Ordenanzas, 41 (5). 
En este pasaje cabe destacar que la causa de la apropiación egoísta es el pecado. Sólo en "policía y concierto de República”, es decir, en una perspectiva de humanismo cívico, se puede cuidar la propiedad común. Además, señala que "los pobres" no sólo son los destinatarios, sino los propietarios mismos de los bienes del hospital-pueblo. Claramente, dentro del hospital no son "pobres" en el tercer sentido de necesidad, y por estar en el mismo, lo son sólo en el sentido de la virtud cristiana, aunque, por lo antes expuesto, no deja de reconocer el peligro de volverse, si están solos, pobres por el pecado y la humana condición de necesidad. Esto nos lleva a subrayar brevemente el último tema: la riqueza de las repúblicas quiroguianas consiste en una propiedad común, en un particular sentido de "comunidad de bienes".

\section{COMUNIDAD DE BIENES}

Cuando hemos hablado de las fuentes de inspiración de Vasco de Quiroga, consideramos como elemento fundamental el ejemplo de las comunidades cristianas que se describen en los Hechos de los apóstoles ${ }^{54}$. El madrigalense está convencido, como ya hemos señalado, de que las comunidades indígenas podrán prosperar bajo este modelo porque están llamados a ser una "nueva iglesia renaciente", equiparable a la clásica edad dorada, pero interpretada desde la naturaleza restituida por la gracia. Para Quiroga, acudir a la Nueva España como evangelizador fue un llamado a todas luces espiritual y la principal razón de su viaje, mucho más perentorio que el mismo llamado del rey ${ }^{55}$.

Ésta debía ser, en esencia, la misión de la iglesia: contribuir a mostrar el camino de la gracia a un pueblo que, admirablemente, ya vivía conforme a este virtuoso modelo. En efecto: Vasco de Quiroga reconoce que los tres citados modelos -la utopía, la edad dorada y la Iglesia-son pertinentes en el Nuevo Mundo porque presentan características que ya eran vividas por las comunidades indígenas antes de la conquista y mediante este sistema podrá contribuir a preservar su modo de vida ${ }^{56}$.

Entre los elementos que reconoce en la cultura indígena y coincide con sus modelos se encuentra el principio de comunidad de bienes que practicaban las primeras comunidades cristianas. Dos pasajes en Hechos de los apóstoles recogen este testimonio: Hechos 2, 44, "Todos los creyentes vivían unidos y tenían todo en común" y Hechos 4, 32, "La multitud de los creyentes no tenía sino un

54 Información en Derecho, III, 241.

55 Cf. Testimonio de Cabrera, \$12, pp. 128-129.

56 Información en Derecho, III, 243-249. 
solo corazón y una sola alma. Nadie llamaba suyos a sus bienes, sino que todo era en común entre ellos" ${ }^{\$ 7}$. En la continuación de ambos pasajes se refiere que las posesiones eran vendidas para repartir el dinero y remediar las necesidades de la comunidad, o que se repartían los bienes según lo que cada quien necesitara, además de compartir el pan.

Conviene, sin embargo, distinguir esta práctica del concepto filosófico de "bien común", aunque en principio tengan la misma raíz. A grandes rasgos puede decirse que el principio de "bien común" parte de una abstracción racional que funciona como criterio para la práctica de la justicia en el sentido lega $1^{58}$. Es una condición de posibilidad de la virtud, más que un bien concreto y es por lo tanto indivisible. En cambio, la comunidad de bienes se refiere a una práctica concreta: el hecho de que los bienes que se tienen sean considerados como propiedad de la comunidad y que todos puedan disponer de ellos a discreción. La base de esta "discreción" es la justicia total, es decir, la comprensión de que la justicia es hacer el bien "al otro", de una forma práctica y concreta, no teórica ni abstracta, y conforme a sus necesidades presentes ${ }^{59}$. Tomás Moro ya señala esta diferencia en la Utopía cuando dice:

En Utopía, como todo es de todos, nunca faltará nada a nadie mientras todos estén preocupados de que los graneros del Estado estén llenos. Todo se distribuye con equidad, no hay pobres ni mendigos y aunque nadie posee nada todos sin embargo son $\operatorname{ricos}^{60}$.

De igual manera, Vasco de Quiroga propone que los hospitales-pueblo estén organizados como una república en que todos los miembros participen, no sólo en las cuestiones de gobierno, sino también en las de producción. Citamos a continuación algunos ejemplos de las Ordenanzas:

(50) Los que viven o vienen a Hospital han de tener los oficios mecánicos y otros útiles necesarios al dicho provecho y bien común del hospital y sus moradores, como son los oficios de tejedores y los otros todos a este oficio anexos y pertenecientes, como son los canteros, carpinteros, albañiles,

57 Biblia de Jerusalén (Bilbao: Desclee de Brouwer, 2009).

58 Tomás de Aquino, STh I-II, q. 98. Ver también la definición en Gaudium Spes n. 26: "el conjunto de aquellas condiciones de la vida social que permiten a los grupos y a cada uno de sus miembros conseguir más plena y fácilmente su propia perfección".

59 Ética nicomaquea V-1, 1129b 30-35. Así pues, una lectura humanista del texto aristotélico traduciría la "koinonía" como una comunidad porque "comunica", es decir, pone en común sus bienes, de hecho. Por eso, es uno de los términos que integran la polémica de Bruni con Cartagena en torno a la traducción filosófica de los términos griegos.

60 Ordenanzas, 75. 
herreros y otros semejantes, (...) de los cuales cada cual de vosotros aprenda el suyo, (...) y no otros vanos, inútiles, curiosos y viciosos.

(52) Además de esto, todos habéis de saber bien hacer y ejercitados y diestros en el oficio de la agricultura desde la niñez, con mucha gana y voluntad, porque ha de ser este oficio de la agricultura común a todos para cada uno cuando y según y cómo se mandare y sea menester que entendáis en él.

(53) Y este trabajo lo hará cada uno respetando las dichas horas de cada día y no más. Podrá salir dos o tres días de trabajo de sol a sol en la semana cada uno poco más o menos, según la necesidad, comodidad, y utilidad del tiempo y de la labor del campo se ofreciere (...).

(55) Por la manera como son los niños (...) deben ser enseñados en la agricultura de la forma siguiente: que después de las horas de doctrina, se ejerciten dos días de la semana en ella, sacándolos su maestro y otro al campo en alguna tierra de las más cercanas a la escuela (...) y esto a manera de regocijo, juego o pasatiempo, una hora o dos cada día, aunque se pierda aquellos días las horas de la doctrina, pues esto también es doctrina y moral de buenas costumbres. Deben ir con sus cosas o instrumentos de la labor que tengan todos para ello, y que de lo que se beneficiaren, sea para ellos mismos. Que se beneficien y cojan todos juntos, para que se enseñen y repartan (...).

(56) Las niñas en las familias de sus padres aprendan los oficios mujeriles que más se les dan a ellas, adaptados y necesarios al provecho y bien suyo $y$ de la república del Hospital, como son obras de lana, lino, seda y algodón, y para todo lo accesorio y necesario y útil al oficio de los telares y juntamente hendan a la vuelta de sus casas y familias.

(57) Lo que de las seis horas del trabajo común se hubiere cogido, se reparta entre vosotros todos y cada uno en partes iguales, congrua, cómoda y honestamente según que cada uno, según su calidad y necesidad, manera y condición, lo haya menester para sí y su familia, de manera que ninguno padezca en el Hospital necesidad ${ }^{61}$.

Puede verse, de esta manera que las diversas funciones se rotan y que los bienes son puestos en común para la satisfacción efectiva de las necesidades de la comunidad misma ${ }^{62}$. Así, no sólo son comunes "los bienes" sino las actividades que los producen, de manera que el fruto de la comunidad de bienes es, sobre todo, la "comunidad misma" que comparte no sólo el pan en la mesa, sino el hecho mismo de sembrar el trigo. Por eso serán importantes para Vasco las

61 Ordenanzas, 50-57.

62 Ordenanzas, 41, 47, pp. 147, 149. 
fiestas y las actividades comunes, además del trabajo: todas son experiencias comunitarias.

Es verdad que Vasco de Quiroga da al trabajo un valor positivo, a diferencia del modelo clásico de la edad dorada. Este valor positivo está presente también en Moro $^{63}$. Remedia la necesidad física, procura la virtud de la generosidad y construye la vida comunitaria, es decir, interviene en los tres tipos de pobreza que consideramos anteriormente. Tampoco se niega la propiedad, sino que se genera y distribuye de manera comunitaria, con unas prácticas que recuerdan lejanamente al concepto de justicia en la república platónica, promoviendo igualmente la riqueza común, la virtud cívica y el desapego individual.

También es cierto que este sistema de trabajo y sustento comunitario había sido vivido por la cristiandad durante siglos en la dinámica de los monasterios. De igual manera, en muchas órdenes religiosas comúnmente se rotan los cargos y se ponen a votación algunos puestos de organización y administración. La pertinencia de la observación subraya la vocación cristiana de la utopía quiroguiana. No obstante, es cierto que, en la redacción de la Información en Derecho, Vasco de Quiroga basa su idea directamente en Moro, y éste a su vez, toma como punto de partida los viajes de Américo Vespucio, de modo que la inspiración de la utopía pareciera venir de América misma, al menos en parte, y por tanto que ésta sea la causa de su idoneidad, como el mismo Quiroga sospecha ${ }^{64}$.

Lo más relevante, sin embargo, es que la inspiración en las comunidades cristianas no consiste en revivir una práctica de antaño, sino reconocer que la naturaleza de los indios ya está dispuesta a las condiciones de virtud que esta práctica entraña y sólo les falta la instrucción cristiana para que comprendan la raíz espiritual de dicha virtud ${ }^{65}$. La instrucción religiosa que deben recibir y después practicar en los hospitales-pueblo está encaminada a hacerles conocer el fundamento teológico y antropológico que entraña la dimensión cristiana y que ni los clásicos latinos o griegos pueden explicar.

A diferencia de la ficción de Tomás Moro, la utopía práctica de Vasco de Quiroga se realizó en Michoacán dentro de una misión de humanismo cristiano, cuya vertiente cívica no deja de vincular al indio, pobre y necesitado del auxilio de la gracia, con el pueblo de Dios. Quizá por esto sea pertinente concluir estas líneas con una declaración última de los objetivos de fundación de los hospitales-pueblo de Vasco de Quiroga:

63 Utopía I-5-3.

64 Información en Derecho, 243.

65 Información en Derecho 241. 
(3) El fin de este Hospital y Colegio de Santa Fe y de su fundador, es que sea un lugar donde, viviendo en concierto y buena policía, no tengáis necesidad, viváis con seguridad y fuera de peligro e infamia de que estáis tan infamados; sin ociosidad; sin codicia desordenada, y sin malas ignorancias y con conocimiento de la doctrina Cristiana (...).

(4) Aquí deseamos que, quitándoles lo malo y dejándoles lo bueno de vuestras costumbres, manera y condición, no vayan a dar en despeñaderos de almas y cuerpos (...) Por eso conservándoles siempre lo bueno que tengan y no destruyéndoselo ni trocándoselo por lo que no les cuadra ni conviene (según suerte y manera de vivir, entendimiento, estado y condición), se respete su calidad, necesidades y capacidad como personas, para que no pierdan lo bueno que tienen de humildad y obediencia, paciencia, poca codicia y buena simplicidad.

(5) (...) También presupone que no les falte la debida y honesta diligencia y prudencia, que os conviene mucho a todos tener cuanto os falta. Por lo que hay que ponerse fuera del peligro de las tres fieras bestias que todo en este mundo lo destruyen y corrompen, que son soberbia, codicia y ambición ${ }^{66}$.

\section{CONCLUSIONES}

Hemos mostrado la presencia de algunos temas de índole económica en la obra y las acciones de Vasco de Quiroga y, asimismo, presentado el espíritu humanista que se refleja en esos temas, principalmente en la pobreza, el trabajo y la comunidad de bienes.

Para ello, hemos señalado brevemente cómo surgió un interés renovado por la economía en el mundo renacentista y cómo perfiló el humanismo sus intereses hacia los temas de la vida práctica, caracterizados por un tenor comunitario que se ha dado en llamar "humanismo cívico". Éste tiene su origen en dos ideas básicas: el hombre necesita desarrollarse como ser moralmente virtuoso y necesita hacerlo en sociedad.

Después hemos expuesto los temas de pobreza, trabajo y comunidad de bienes. Sobre la pobreza, hemos considerado el clima conceptual complejo en torno al problema de la pobreza en el tiempo de Vasco de Quiroga, teniendo como parámetro comparativo la obra de Juan Luis Vives. De este análisis han surgido tres dimensiones de la pobreza: la antropológica u originaria, la moral y la 
propiamente económica o material (las cuales no son excluyentes entre sí). Adicionalmente, se mostró el modo en que los tres conceptos se aplican a la idea de "pobreza" con la que se relaciona la obra de Vasco de Quiroga.

Defendimos que el trabajo constituye una vía práctica de mejoramiento humano y con respecto a la comunidad de bienes; sobre esta idea, la hemos distinguido del concepto de "bien común" y hemos mostrado cómo la comunidad de bienes es un hecho concreto y realizable como justicia comunitaria.

Ambos medios, el trabajo y la comunidad de bienes, son remedios efectivos contra los tres tipos de pobreza. Contra la pobreza económica, por proveer de medios para la subsistencia, tanto para el individuo como para la comunidad; contra la pobreza moral, porque mediante el trabajo se evitan los vicios del ocio y la pereza, con la generosidad comunitaria se preservan la humildad, el desapego y la solidaridad propias de la vida cristiana; finalmente, contra la pobreza de la condición humana, porque mediante el trabajo el individuo crece y mejora en virtud y lo hace comunitariamente porque no puede desarrollarse la virtud en soledad: la vida virtuosa es un asunto cívico.

\section{REFERENCIAS BIBLIOGRÁFICAS}

\section{FUENTES PRIMARIAS}

Aquino, Tomás de. Suma Teológica, I-II. Madrid: BAC, 2011.

Moro, Tomás. Ordenanzas. Editado por Guillermo Rovirosa. Madrid, editorial ZYX, 1964.

Quiroga, Vasco de. Información en Derecho. Editado por de Carlos Herrejón Peredo. México: Cien de México-SEP, 1985.

- . "Reglas y Ordenanzas para el gobierno de los hospitales de Santa Fe en México y Michoacán". En Fragmentos de la vida y virtudes del Varón Ilustrísimo y Reverendísimo, Sr. Dr. Don Vasco de Quiroga, Primer obispo de la Santa Iglesia Catedral de Michoacán y fundador del Real y Primitivo colegio de San Nicolás Obispo de Valladolid, editado por Juan Joseph Moreno. México: Talleres Gráfico de la Nación, 1940.

- . "Reglas y Ordenanzas para el gobierno de los hospitales de Santa Fe en México y Michoacán”. En Vasco de Quiroga, jurista con mentalidad secular, editado por Pablo Arce Gargollo. México: Porrúa-Universidad Panamericana, 2007.

-. "Testamento", En Vasco de Quiroga, jurista con mentalidad secular, editado por Pablo Arce Gargollo. México: Porrúa-Universidad Panamericana, 2007. 


\section{BIBLIOGRAFÍA SECUNDARIA}

Aguayo Spencer, Rafael. Don Vasco de Quiroga. Pensamiento jurídico, Antología. México: Porrúa, 1979

Arce Gargollo, Pablo. Vasco de Quiroga, jurista con mentalidad secular. México: Porrúa-Universidad Panamericana, 2007.

Arriaga, Antonio. "Vasco de Quiroga, fundador de pueblos". Estudios de Historia Novohispana, 1-1(1966).

http://www.revistas.unam.mx/index.php/ehn/article/view/-3196/2751.

Consultado el 15 de abril de 2019.

Barrientos García, José. Repertorio de moral económica (1526-1670), La Escuela de Salamanca y su proyección. Pamplona: EUNSA, 2011.

Campos, Carlos Alberto. "Carlos V, Tomás Moro y Vasco de Quiroga". XXII Coloquio de Historia Canario-Americana (2016) XXII-072, 1-11. http://coloquioscanariasmerica.casadecolon.com/index.php/aea/article/view/10017. Consultado el 15 de abril de 2019.

Casado Arboniés, Manuel, "Real Provisión de los Reyes Católicos ordenando establecer en la ciudad de Sevilla una Casa de Contratación y promulgando las Ordenanzas por las que ha de regirse". Consulta electrónica: http://www3.uah.es/cisneros/carpeta-/images/pdfs/253.pdf. Consultado el 3 de abril de 2019.

Cuevas, Mariano. Historia de la Iglesia en México, Tomo I, 1511-1548. México: Patria, 1946.

Garin, Eugenio. El renacimiento italiano. Barcelona: Ariel, 1986.

Grice-Hutchinson, Marjorie. The School of Salamanca: Readings in Spanish Monetary Theory, 1544-1604. Ludwig Von Mises, Auburn, 2011.

Herrejón Peredo, Carlos "Introducción”. En Información en derecho de Vasco de Quiroga. Ed. Secretaría de Educación Pública, Col. Cien de México, México, 1985.

Ladero Quesada, Miguel Ángel. El primer oro de América: los comienzos de la casa de contratación de las Yndias, 1503-1511. Madrid: Real Academia de Historia, 2002.

León, Nicolás. El Ilustrísimo señor don Vasco de Quiroga, primer obispo de Michoacán. Grandeza de su persona y de su obra. México: Sucesores de F. Díaz de León, 1903.

Méndez Alonso, Manuel. "La discusión sobre Vita Activa, Nobilitas y Libertad en la Utopía de Tomás Moro". Revista Portuguesa de Filosofía 67, no. 2 (2011): 355-376.

Miranda, Francisco. Vasco de Quiroga, Varón universal. México: Jus, 2007.

Moreno, Juan José. Fragmentos de la vida y virtudes del Ilustrísimo don Vasco de Quiroga. México: Imprenta del Real y más Antiguo Colegio de San Ildefonso, 1766. 
Pérez Priego, Miguel Ángel, "Encuentro del viajero Pero Tafur con el humanismo florentino del primer cuatrocientos". Revista de Literatura, LXXIII, n. 145 (enero-junio 2011): 131-142.

Sabido, Cecilia. "El ideal humanista de mejoramiento humano y su influencia en Vasco de Quiroga". Metafísica y persona 21 (2019):183-197.

- . "El programa humanista en la utopía práctica de Vasco de Quiroga". En Tomás Moro y Vasco de Quiroga, Utopías en América, 115-135, editado por Virginia Aspe. Pamplona: Pensamiento Medieval y Renacentista EUNSA, 2018:

Sebastián Solanes, Raul Francisco. "La aportación de Juan Luis Vives al estudio de la pobreza. Del socorro de los pobres a la aporofobia". Vivesiana 3 (2018): 75-87.

Valero Moreno, Juan Miguel. "Antecedentes y encrucijadas de la vida activa y contemplativa en la Castilla del Cuatrocientos". eHumanista 29 (2015): 3271.

Virgilio Marón, Publio et al. Bucólicas, Geórgicas, Apéndice virgiliano. Traducido por T. Recio García. Madrid: Gredos, 1990.

Vives, Juan Luis. "Tratado del socorro de los pobres". Traducido por Juan de Gonzalo Nieto. En Clásicos de Historia. Compilado por J. Martínez. http://clasicoshistoria.blogspot.com/2017/09/juan-luis-vives-tratado-delsocorro-de.html (Consultado el 3 de abril de 2019)

Zavala, Silvio. Ideario de Vasco de Quiroga. México: Colegio de México, 1941.

—. Recuerdo de Vasco de Quiroga. México: Porrúa, 2007.

Cecilia Guadalupe Sabido Sánchez Juárez Facultad de Ciencias Sociales y Humanidades

Universidad Autónoma de San Luis Potosí Av. Industrias 101-A, Fracc. Talleres 78399, San Luis Potosí, SLP, (México) https://orcid.org/0000-0002-6028-4813

Élida María Tedesco

Facultad de Ciencias Sociales y Humanidades Universidad Autónoma de San Luis Potosí Av. Industrias 101-A, Fracc. Talleres 78399, San Luis Potosí, SLP, (México) https://orcid.org/0000-0003-0314-2680 